ISSN: $1130-3743$

\title{
EL APRENDIZAJE EN LA UNIVERSIDAD Y LA TEORÍA DEL PROCESO DUAL DE RAZONAMIENTO ${ }^{1}$
}

\author{
Learning in university and dual process theory \\ of reasoning
}

\section{L'apprentissage sur l'université et la théorie du double processus de raisonnement}

\author{
Miguel López AsTORGA \\ Universidad de Talca. Instituto de Estudios Humanísticos "Juan Ignacio Molina". \\ Av. Lircay, s/n. Talca (Chile).Correo-e: milopez@utalca.cl
}

Fecha de recepción: marzo de 2013

Fecha de aceptación: junio de 2013

Biblid [(1130-3743) 25, 2-2013, 231-246]

RESUMEN

En este trabajo, argumentamos que existen relaciones entre dos tipos de aprendizaje, el aprendizaje asociativo y el aprendizaje comprensivo, y los dos sistemas de razonamiento propuestos por la teoría dual. En concreto, tratamos de mostrar que el aprendizaje asociativo se encuentra vinculado con el Sistema 1 y que el aprendizaje

1. Este artículo es resultado del proyecto $n .^{\circ} 1120007$, «El procesamiento de enunciados condicionales en los estudiantes de Educación Media: un estudio a partir de la tarea de selección de Peter Wason", del Fondo Nacional de Desarrollo Científico y Tecnológico (FONDECYT), de la Comisión Nacional de Investigación Científica y Tecnológica (CONICYT), Ministerio de Educación, Gobierno de Chile. El autor, que es el investigador responsable del mencionado proyecto, agradece al programa FONDECYT y a las instituciones nombradas el financiamiento de este trabajo. 
comprensivo hace referencia al Sistema 2. Al mismo tiempo, revisamos críticamente la tesis de Pérez, Soto, Sola y Serván (2009) relativa a favorecer el desarrollo del aprendizaje comprensivo, y, por tanto, del Sistema 2, y minimizar la acción del aprendizaje por asociación, y, por tanto, del Sistema 1. En nuestra opinión, el aprendizaje asociativo (Sistema 1) también es necesario para el conocimiento humano y, por esta razón, concluimos que debe ser revisado y analizado por medio del aprendizaje comprensivo (Sistema 2), pero no rechazado.

Palabras clave: aprendizaje comprensivo, aprendizaje por asociación, conocimiento humano, teoría dual de razonamiento.

\section{SUMMARY}

In this paper, I argue that there are relationships between two kinds of learning, associative learning and comprensive learning, and the two reasoning systems described by dual process theory. Specifically, I try to show that associative learning is linked to System 1 and comprehensive learning is related to System 2. At the same time, I review critically the Pérez, Soto, Sola and Serván (2009)'s thesis in favour of the development of comprehensive learning, and, therefore, of System 2, and of the minimization of associative learning, and, therefore, of the System 1. In my opinion, associative learning (System 1) is necessary for human knowledge and, for this reason, I conclude that it should be analyzed through comprehensive learning (System 2), but not rejected.

Key words: associative learning, comprensive learning, dual process theory of reasoning, human knowledge.

\section{SOMMAIRE}

À ce travail, nous arguons que des relations existent entre deux types d'apprentissage, l'apprentissage associatif et l'apprentissage compréhensif, et les deux systèmes de raisonnement proposés par la théorie dual. En somme, nous essayons de montrer que l'apprentissage associatif se trouve lié avec le Système 1 et que l'apprentissage compréhensif fait référence au Système 2. En même temps, nous révisons de façon critique la thèse de Pérez, Soto, Sola et Serván (2009) relative à favoriser le développement de l'apprentissage compréhensif, et, donc, du Système 2, et minimiser l'action de l'apprentissage par association, et, donc, du Système 1. Selon notre opinion, l'apprentissage associatif (le Système 1) est aussi nécessaire pour la connaissance humaine et, par cette raison, nous concluons qu'il doit être révisé et être analysé au moyen de l'apprentissage compréhensif (le Système 2), mais non repoussé.

Mots clés: apprentissage compréhensif, apprentissage par association, connaissance humaine, théorie dual de raisonnement. 


\section{INTRODUCCIÓN}

Una gran preocupación se observa, a nivel mundial, por mejorar la educación universitaria. El proceso de Bolonia en Europa significa, sin duda, un intento en este sentido que queda recogido, por ejemplo, en Pérez, Soto, Sola y Serván (2009). Pérez y sus colaboradores defienden, entre otros interesantes planteamientos, que el aprendizaje que debe presidir en las instituciones de educación superior es el aprendizaje comprensivo, y no el aprendizaje por asociación o pragmático.

El primero de estos tipos de aprendizaje apunta a la racionalidad, a la cientificidad, a la significatividad, al análisis y a la reflexión consciente. Sin embargo, el segundo hace referencia a la rapidez, a la rutina y, casi, a la inconsciencia. De los planteamientos de Pérez et al. (2009), podemos deducir que el aprendizaje por asociación parece natural en los seres humanos y que es el que predomina en los estudiantes que ingresan en las universidades. La labor docente, por tanto, debe consistir en modificar ese aprendizaje que caracteriza a los estudiantes por un aprendizaje comprensivo que se fundamente en la ciencia y que pueda ser revisado y discutido.

Estas pretensiones de Pérez et al. (2009) parecen consistentes con una teoría relativamente reciente en el área de la ciencia cognitiva y que, en el momento presente, con matices y ciertas discrepancias menores, es aceptada casi unánimemente en sus rasgos esenciales por los teóricos de dicho ámbito. Nos referimos a la teoría dual de razonamiento, defendida o descrita, entre otros trabajos, en Evans (1989, 2006, 2008), Stanovich (1999, 2004), Reyna (2004) y López (2009a).

En líneas generales, la teoría dual de razonamiento defiende que existen dos sistemas diferentes de conocimiento. Estos dos sistemas han recibido diferentes nombres, pero, según entendemos, la terminología que se ha popularizado y generalizado es la empleada por Stanovich (1999, 2004), quien se refiere a ellos como Sistema 1 y Sistema 2. El Sistema 1 es habitualmente descrito como una instancia cognitiva encargada del ámbito intuitivo y que opera por heurísticos, ya sean éstos innatos o adquiridos por la experiencia. Cuando utiliza el Sistema 1, el individuo actúa deprisa y de manera apenas consciente. Por su parte, el Sistema 2 presenta características diametralmente opuestas. El Sistema 2 tiene que ver con el razonamiento inferencial y con el análisis, y, cuando es empleado por el sujeto, demanda tiempo y cierto esfuerzo consciente.

Desde nuestro punto de vista, existe un paralelismo evidente entre los dos tipos de aprendizaje comentados por Pérez et al. (2009) y los dos sistemas cognitivos de la teoría dual de razonamiento. En este trabajo, vamos a tratar de demostrar que, efectivamente, tal paralelismo es real y que, a partir de él, es posible extraer interesantes consecuencias pedagógicas, como, por ejemplo, que la reivindicación de Pérez y sus colaboradores relativa a la necesidad de potenciar en la Universidad el aprendizaje comprensivo puede significar, en la práctica, un desarrollo de las capacidades implicadas en el Sistema 2, en detrimento de las 
presentes en el Sistema 1. No obstante, lo primero es, quizás, mostrar las relaciones que pueden observarse entre, por una parte, el Sistema 1 y el aprendizaje por asociación y, por otra, el Sistema 2 y el aprendizaje comprensivo. Comencemos, pues, con tal labor.

\section{El APRENDIZAJE PRAGMÁtiCO POR ASOCIACIÓN Y EL SiSTEMA 1}

Siguiendo a Wasserman y Miller (1997), se puede decir que la investigación científica acerca del aprendizaje asociativo comenzó hace más de un siglo, con estudios como los de Thorndike y Pavlov, y que este tipo de aprendizaje continúa siendo en la actualidad un tema sobre el que se trabaja muy activamente. Estos autores plantean que el aprendizaje asociativo debería ser considerado el fundamento para comprender formas de comportamiento y de cognición en humanos y animales. Sin embargo, Pérez et al. (2009) parecen preferir el concepto de aprendizaje pragmático por asociación que se deriva de propuestas como las de Lave y Wenger (1991) y Reber (1993). Como es sabido, Lave y Wenger (1991) propusieron que el aprendizaje no debe ser entendido como lo es tradicionalmente y que debe ser superada la concepción que lo considera, básicamente, como un proceso de adquisición de datos y de información. Desde su óptica, se hace preciso atender a todas las dimensiones implicadas en el proceso de aprendizaje y no olvidar las estrechas relaciones que mantiene el individuo que aprende con su entorno y con las actividades prácticas que realiza dentro de una comunidad.

Por su parte, Reber (1993) apunta a aquellos aprendizajes en los que el resultado parece emerger sin la necesidad de mayores esfuerzos, esto es, a aquellos aprendizajes que se vinculan, en su opinión, con el problema del conocimiento tácito, ya estudiado, según nos parece indicar, por la filosofía y por autores como Wittgenstein, Husserl, Putnam o Michael Polanyi. A su juicio, los aprendizajes más esenciales, como el de la lengua natural o el referente al desarrollo de capacidades para la socialización, se pueden relacionar con procesos de este tipo, los cuales son caracterizados por él como implícitos.

Puede ser, no obstante, también esclarecedora la explicación que, desde un punto de vista lingüístico, nos ofrece Ellis (2011) al respecto. Para Ellis (2011), los niños comienzan a adquirir el lenguaje mediante su participación en una comunicación natural coherente con las personas que los cuidan. Así, automáticamente, van disponiendo del conocimiento que necesitan de la estructura de su idioma. Lo paradójico, desde su punto de vista, es que ellos no pueden describir este conocimiento, hasta el punto de que, si le preguntamos a un niño pequeño que utilice correctamente el plural en su lengua en conversaciones cotidianas cómo se forma el plural, cabe la posibilidad de que nos responda que no sabe hacerlo. Ello se debe, según entendemos, a que su aprendizaje del uso del idioma se encuentra relacionado con el aprendizaje asociativo y la explicitación de las reglas gramaticales con el comprensivo. 
Pero en Pérez et al. (2009) estas formas de aprendizaje no parecen ser valoradas muy positivamente, siendo, además, muy interesante para este trabajo que se relacionan con una serie de características que coinciden, en gran medida, con las que asignan los defensores de la teoría dual de razonamiento al Sistema 1. Procedemos a revisar, en los párrafos siguientes, cómo describen Pérez et al. (2009) el aprendizaje asociativo y cuáles son las relaciones que se pueden establecer entre tal descripción y las propiedades del Sistema 1.

El aprendizaje por asociación se presenta como un aprendizaje necesario y útil para poder orientarse y desenvolverse en la vida cotidiana, pero que, sin embargo, es mínimamente fiable, poco riguroso y aproximativo. Estamos ante un aprendizaje "implícito y experiencial" (Pérez et al., 2009, 7) y "frecuentemente mecánico, rutinario, y por ello el conocimiento que produce suele estar cargado de lagunas, prejuicios e inexactitudes" (Pérez et al., 2009, 7).

Estas características no se hallan muy distantes de las que nos ofrece para el Sistema 1 Evans (2008) en sus tablas descriptivas de los dos sistemas de la teoría dual de razonamiento. Evans (2008) nos recuerda que es Reber (1993), autor citado, como hemos indicado, por Pérez y sus colaboradores para fundamentar su concepto de aprendizaje asociativo, el que se refirió al Sistema 1 como un sistema implícito (implicit), así como también que Epstein (1994) lo denominó experiencial (experiential). Por lo demás, otras características que Evans (2008) enumera también encajan perfectamente, del mismo modo, con la cita de Pérez et al. (2009) que hemos reproducido. Evans indica que el Sistema 1 es automático (automatic), adjetivo que no parece estar muy lejos de mecánico, término empleado por Pérez y sus colaboradores, y que en él es posible el proceso defectuoso (default process), idea compatible con la de las lagunas, los prejuicios y las inexactitudes que, según Pérez et al. (2009) acompañan al aprendizaje asociativo.

Pero, por si esto no fuera suficiente, tenemos también que Evans (2008) asigna igualmente al Sistema 1 las propiedades utilizadas para nombrar esta forma de aprendizaje que Pérez y sus colaboradores consideran propia del alumnado que ingresa a la Universidad y que debería erradicarse, puesto que señala que el Sistema 1 es asociativo (associative) y pragmático (pragmatic).

Por tanto, si aceptamos la correspondencia entre el aprendizaje pragmático por asociación y el Sistema 1, podemos interpretar que las tesis de Pérez et al. (2009) implican que los estudiantes que llegan a la Universidad poseen aprendizajes que son fundamentalmente el producto del Sistema 1 y que el objetivo de las instituciones de educación superior debe ser reemplazar tales aprendizajes por otros que sean el resultado del Sistema 2. Empero, para confirmar aún más esta interpretación nuestra, vamos a revisar en el apartado siguiente las características del aprendizaje comprensivo para comprobar si también es posible encontrarlas en las descripciones que ofrece la ciencia cognitiva del Sistema 2. 


\section{El APRENDIZAJE COMPRENSIVO Y EL SISTEMA 2}

En opinión de Pérez y sus colaboradores, "en la universidad, así como en las etapas anteriores de la vida escolar de cada ser humano, lo que se pretende es provocar un aprendizaje comprensivo [en el texto original, los autores escriben aprendizaje comprensivo con negritas], significativo, científico, explícito, discutible y fundamentado" (Pérez et al., 2009, 8). Por consiguiente, este otro tipo de aprendizaje apunta al desarrollo de capacidades analíticas y reflexivas propias de las formas de saber que habitualmente consideramos ciencia. La idea de Pérez y sus colaboradores parece ser, así, sustituir el conocimiento cotidiano de los estudiantes por un conocimiento bien fundamentado, basado en evidencias objetivas, que detente un cierto grado de rigor y que permita extraer conclusiones válidas desde el punto de vista lógico.

Quizás, puede decirse que, desde el enfoque lingüístico de Ellis (2011), este tipo de aprendizaje corresponde al momento en el que el individuo ya no sólo sabe utilizar perfectamente su idioma, sino que también conoce sus reglas y es capaz de analizarlas y de reflexionar acerca de ellas. No obstante, lo relevante aquí parece ser que esta descripción que acabamos de exponer del aprendizaje comprensivo parece ser totalmente compatible con las características que en la literatura de la ciencia cognitiva se le atribuyen generalmente al Sistema 2. Como queda expuesto en las tablas de Evans (2008), el Sistema 2 es frecuentemente descrito como consciente, explícito, controlado, analítico, reflexivo, basado en reglas, lógico...

En este sentido, creemos digna de mención la labor de sistematización que realiza Evans (2008), quien en sus tablas nos recuerda, entre otros datos de suma relevancia, que Schneider y Schiffrin (1977) consideraron al Sistema 2 controlado (controlled), que Epstein (1994) lo denominó racional (rational), que Reber (1993) hizo referencia a él como un ámbito explícito (explicit), que él mismo en trabajos anteriores (por ejemplo, Evans, 1989) pensó en este sistema como un sistema analítico (analytic), que Sloman (1996) se centró en el hecho de que se encuentra basado en reglas (rule based), que Wilson (2002) insistió en su carácter consciente (conscious)...

Ante este elenco de autores y de atributos correspondientes al Sistema 2, pocas dudas albergamos de la conexión inequívoca que existe entre lo que Pérez y sus colaboradores denominan aprendizaje comprensivo y el Sistema 2 de la teoría dual de razonamiento. Hemos de tener en cuenta que la enumeración de autores que aportan características para el Sistema 2 podría ser mucho más extensa, como nos lo demuestra la extraordinaria labor de Evans (2008). No obstante, creemos que los nombrados son lo suficientemente representativos como para fundamentar la relación entre tipos de aprendizaje y sistemas de conocimiento que estamos defendiendo desde el comienzo de este trabajo. Lo interesante ahora es, desde nuestro punto de vista, extraer las conclusiones que pueden derivarse de la supremacía que Pérez et al. (2009) atribuyen al aprendizaje comprensivo. 


\section{El Sistema 2 Y SU PROBLEMÁticA}

Evidentemente, como hemos apuntado más arriba, las consecuencias teóricas más inmediatas que, tras nuestra comparación con la teoría dual de razonamiento, se derivan del enfoque de Pérez et al. (2009) hacen referencia a la necesidad de trabajar con los alumnos universitarios su Sistema 2, minimizando, en la medida de lo posible, la incidencia que pueda tener en su cognición el Sistema 1. Y es que, tras los vínculos que hemos hallado entre el aprendizaje comprensivo y el Sistema 2, parece obvio que, si deseamos un tal aprendizaje comprensivo, sólo queda fomentar la acción del Sistema 2 del alumnado. La actividad del Sistema 1, como decimos, debe ser, si no ignorada, desactivada hasta cierto punto, ya que sus productos suelen ser inexactos y los conocimientos que nos proporciona a menudo se encuentran repletos de vacíos de cierta relevancia.

Así, podemos vislumbrar más elementos que nos autorizan al establecimiento de nuestro paralelismo entre los tipos de aprendizaje de Pérez y sus colaboradores y los sistemas de la teoría dual. Por ejemplo, desde la teoría dual se sostiene que el Sistema 2 debe revisar y, si es preciso, corregir las intuiciones del Sistema 1 (sobre este asunto, véanse, por ejemplo, Inglis y Simpson, 2006, o López, 2009a), algo que parece perfectamente compatible con la idea de Pérez et al. (2009) relativa a que el aprendizaje comprensivo debe brotar como resultado de una reelaboración de los conocimientos adquiridos de modo pragmático.

No obstante, el problema reside, a nuestro juicio, en el hecho de que, a partir de esta correspondencia entre la propuesta de Pérez y sus colaboradores y la teoría dual, parecen revelarse ciertos supuestos que precisan ser analizados y comentados. Una primera dificultad parece encontrarse en que, si realizamos una lectura apresurada del trabajo de Pérez y sus colaboradores, podemos concluir que ellos piensan que el aprendizaje comprensivo es necesario en la Universidad porque en las etapas educativas anteriores el aprendizaje es más de tipo asociativo y pragmático y, por consiguiente, se halla centrado en el Sistema 1. Afortunadamente, esta conclusión se muestra como errónea cuando leemos con detenimiento las tesis de Pérez et al. y descubrimos que, como en una cita que hemos reproducido en líneas anteriores, ellos indican explícitamente que el aprendizaje comprensivo y, por consiguiente, el desarrollo de las capacidades implicadas en el Sistema 2, debe caracterizar no sólo a la enseñanza que se imparte en las universidades, sino también a la que se halla presente en los periodos educativos previos.

Sin embargo, distinto es el caso de otro planteamiento derivable mediante el paralelismo que hemos establecido. Si el Sistema 2 es el responsable del razonamiento analítico y lógico, Pérez y sus colaboradores están reivindicando, como hemos señalado, que este tipo de razonamiento, que tan próximo se encuentra al ámbito matemático, es el que prioritariamente se debe fomentar en la educación de los estudiantes. Un enfoque de esta índole inmediatamente nos trae a la mente propuestas en la línea de, por ejemplo, Inhelder y Piaget (1958) o Beth y Piaget (1966), que, de un modo más o menos manifiesto, consideran a lo lógico- 
formal como el estadio madurativo superior que puede ser alcanzado a través del desarrollo cognitivo.

Los vínculos entre las propuestas de corte piagetiano y las tesis de Pérez et al. (2009) parecen evidenciarse con mayor claridad cuando proponen, como estrategias metodológicas para lograr el aprendizaje comprensivo, dos técnicas que tienen en común su aplicabilidad en contextos diversos y en escenarios cambiantes: el contraste de perspectivas y la experimentación del cambio. Más allá de las diferencias entre estas dos estrategias y de su significado real para la práctica educativa, lo interesante para nosotros aquí es que son dos técnicas que podríamos considerar formales, en el sentido de que implican enfrentar al estudiante con situaciones variables y con diferentes maneras de entender una realidad. Parece, por consiguiente, que lo fundamental para Pérez y sus colaboradores es que el alumnado adquiera competencias formales aplicables a cualquier escenario, sean sus características las que sean, prescindiendo de conexiones directas con contenidos concretos, o, al menos, rebajando la trascendencia de tales conexiones.

Pero los enfoques lógico-formales de índole piagetiana han sido, como sabemos, bastante cuestionados, tanto explícita como implícitamente, en los últimos tiempos, pues se ha defendido que, por ejemplo, los heurísticos tienen en la mente humana un papel tan significativo o más que el puro razonamiento lógico. Es interesante en este sentido la discusión que podemos encontrar en López (2008) acerca de la famosa paradoja de los cuervos negros de Hempel (1945), la cual reproducimos a continuación a título meramente ilustrativo.

Hempel (1945) planteó que, para comprobar si todos los cuervos son negros, desde un punto de vista lógico estricto, sería indiferente revisar cuervos para intentar determinar si efectivamente son negros o examinar objetos no negros del universo con el propósito de verificar que no son cuervos. Los dos caminos serían legítimos para el ser humano si él operara mentalmente sólo con un sistema prescriptivo y normativo como puede ser el de la lógica clásica. Empero, para la mayor parte de los seres humanos, analizar objetos no negros para comprobar si son cuervos es una actividad absurda si nuestra tarea es investigar si los cuervos son negros.

Según López (2008), esto es una prueba evidente de que en la mente humana, además de la lógica, intervienen otros factores. Desde su punto de vista, lo que acontece con la paradoja de Hempel puede explicarse gracias a la teoría de la relevancia de Sperber y Wilson (1986). En opinión de Sperber y Wilson, hay dos conceptos fundamentales a tener en cuenta si se desea comprender verdaderamente la dinámica cognitiva humana: efecto cognitivo y esfuerzo cognitivo. El efecto cognitivo se relaciona con las repercusiones que para nuestras creencias puede tener una nueva información. Si ésta nos proporciona creencias novedosas o provoca que rechacemos creencias que considerábamos correctas, tiene efectos cognitivos y, por tanto, la juzgamos como relevante. Por su parte, el esfuerzo cognitivo hace referencia a la intensidad y la cantidad de las operaciones que tenemos que realizar. Si, para asimilar una información determinada, tenemos 
que realizar un gran esfuerzo, esa información no será considerada relevante por nosotros. Así, para que una información sea relevante, ésta debe aportar efectos cognitivos y no requerir mucho esfuerzo cognitivo.

Este planteamiento es, precisamente, el que, desde el punto de vista de López (2008), explica por qué preferimos, para comprobar si todos los cuervos son negros, revisar cuervos a revisar objetos no negros. Atender exclusivamente al conjunto de cuervos aporta mayores efectos cognitivos, ya que cada cuervo nos da información sobre el ave que estamos considerando y los objetos no negros no necesariamente lo hacen (un objeto no negro puede ser, por ejemplo, un lápiz verde), y nos demanda bastante menos esfuerzo cognitivo, pues, si sólo revisamos cuervos, nuestras operaciones mentales no serán tan extenuantes como si analizamos todos los objetos no negros con los que nos encontremos.

Ante estos argumentos, nos resulta obvio que para el conocimiento humano son necesarios elementos no relacionados directamente con el razonamiento analítico y con el Sistema 2, sino con ciertos mecanismos, como los postulados por la teoría de la relevancia, que se encuentran mucho más próximos a los heurísticos y a las intuiciones del Sistema 1. La pregunta es, por tanto, si debemos conceder de manera absoluta, como parecen pretender Pérez et al., la primacía al Sistema 2, siendo éste el que exclusivamente habría que potenciar en los estudiantes, o si, por el contrario, también debemos prestar atención al funcionamiento de factores y ámbitos implicados en el Sistema 1, los cuales pueden desempeñar, como acabamos de comprobar con el ejemplo de la paradoja de Hempel, un rol relevante y fundamental en la cognición humana, al facilitarla y conducirnos a ella con mayor rapidez.

Ciertamente, después de lo expuesto, no parece lo más aconsejable minusvalorar la función del Sistema 1 en el conocimiento. No obstante, en la literatura de la ciencia cognitiva encontramos muchos más ejemplos de las ventajas y beneficios que este sistema le ofrece al ser humano, ejemplos que cuestionan en mayor medida la pretensión de centrar la actividad educativa principalmente en el Sistema 2. A continuación, revisamos algunos de estos ejemplos.

\section{El Sistema 1 y SUS POTENCIALIDADES}

El Sistema 1 parece ser tremendamente útil tanto para la vida cotidiana como para orientar el conocimiento científico. En López (2009a) se nos informa de cómo es posible interpretar, a partir de trabajos como el de Inglis y Simpson (2006), que, gracias al Sistema 1, los campeones de ajedrez pueden jugar partidas paralelas con un considerable número de aficionados al mismo tiempo. Lo pueden hacer porque no necesitan razonar para realizar sus jugadas. Han adquirido heurísticos y destrezas intuitivas en su Sistema 1 que les permiten seleccionar movimientos de un modo rápido y casi inconsciente.

Por otra parte, el comportamiento real de los médicos en ejercicio, estudiado, por ejemplo, por Reyna y Adam (2003), por Reyna, Lloyd y Brainerd (2003) y 
EL APRENDIZAJE EN LA UNIVERSIDAD Y LA TEORÍA DEL PROCESO DUAL DE RAZONAMIENTO

por Reyna (2004), parece encontrarse más guiado por el Sistema 1 que por el Sistema 2. Como nos cuenta Reyna (2004), los médicos suelen interesarse más por la probabilidad de enfermedad coronaria de sus pacientes que por su riesgo de padecer un ataque cardiaco, debido a que, aunque es posible, no es frecuente que alguien padezca un ataque cardiaco sin presentar probabilidades de enfermedad coronaria. Según Reyna, en experimentos sobre este asunto,

\begin{abstract}
Physicians apparently estimated each risk by thinking of the gist of that class of patients: The typical coronary diesease patient is not at risk of an imminent heart attack, but the typical patient at risk of a heart attack has coronary disease [Los médicos aparentemente estimaron cada riesgo pensando en lo esencial de cada tipo de pacientes: el típico paciente con enfermedad coronaria no está en riesgo de un ataque cardiaco inminente, pero el típico paciente en riesgo de un ataque cardiaco padece una enfermedad coronaria] (Reyna, 2004, 62).
\end{abstract}

Puede pensarse que, gracias a estos heurísticos adquiridos a partir de su experiencia laboral ejerciendo la medicina, los médicos realizan su trabajo de un modo más dinámico y pueden atender a un número mayor de pacientes en menos tiempo. Parece ocurrirles algo semejante al proceso que experimentamos cuando aprendemos, en el área de las matemáticas, a aplicar un algoritmo. Al principio, requiere de nuestras habilidades analíticas de razonamiento (Sistema 2), pero, a medida que practicamos el uso de tal algoritmo, podemos emplearlo de un modo más mecánico, automático, rutinario y casi inconsciente (Sistema 1). Aquí la dirección del aprendizaje parece ser la contraria a la deseada por Pérez et al. (2009), pues, en vez de transitar del Sistema 1 al Sistema 2, comienza por el Sistema 2 y finaliza en el Sistema 1.

Como hemos indicado, exiten diversos ejemplos en la literatura de la ciencia cognitiva a los que podemos recurrir para mostrar las virtualidades del Sistema 1. Sin embargo, con el propósito de no extendernos demasiado innecesariamente, vamos a hacer referencia únicamente a uno más que es considerado por algunos (Inglis y Simpson, 2006) como de los más representativos de la teoría dual de razonamiento. Se trata del famoso y prolijamente estudiado problema de Linda (Tversky y Kahneman, 1983). En este problema, se le describe al participante a Linda cuando era estudiante y se le cuenta que era una activista que luchaba contra las discriminaciones y las injusticias en la sociedad. A continuación, se le pregunta cuál cree que podría ser la situación de Linda en la actualidad, cuando cuenta con treinta años de edad. Generalmente, se le ofrecen al participante varias opciones para que las ordene de mayor a menor probabilidad, pero las más relevantes suelen ser dos semejantes a éstas: (A) Linda trabaja como cajera en un banco y (B) Linda trabaja como cajera en un banco y es una activista feminista.

Se pueden encontrar diversas descripciones en español de este problema y de las dificultades que lo acompañan, por ejemplo, en López (2009b) o en López (2011), pero lo que nos interesa de este ejercicio en este trabajo es, principalmente, que un sujeto que sólo extrajera conclusiones por medio de su razonamiento 
lógico-matemático (Sistema 2) tendría forzosamente que decantarse por la opción (A), ya que (B) es un acontecimiento compuesto y un acontecimiento compuesto no puede ser nunca, según la teoría de la probabilidad, más probable que uno de los acontecimientos simples que lo componen, en este caso, (A). No obstante, la mayor parte de los sujetos que realizan esta tarea de razonamiento suele inclinarse, como es previsible, por la alternativa (B), y ello, según parece, porque, al margen de lo establecido por las leyes probabilísticas, los seres humanos piensan en lo más plausible en función de las características de las personas y de las situaciones. Tversky y Kahneman (1983) consideraron que los seres humanos recurren, en circunstancias como ésta, a un heurístico que denominaron heurístico de la representatividad. La acción de este heurístico es la que, en su opinión, conduce al individuo a preferir (B) antes que (A), puesto que le hace juzgar que (B) describe una situación más representativa de la personalidad de Linda. Naturalmente, utilizar el heurístico de la representatividad es recurrir al Sistema 1, sistema que, en último término, es el que permite que nos centremos en la opción que, aparentemente, es más posible para Linda, y no en la indicada por el razonamiento lógico-matemático.

Como podemos apreciar, el Sistema 1 nos aporta una serie de ventajas cognitivas que nos permiten trabajar, como hemos señalado, con mayor celeridad y simplificar procesos. Sin embargo, también entraña peligros y riesgos, lo cual se traduce en que la posición de Pérez et al. (2009) tiene, por supuesto, igualmente, su sentido. Creemos que la manera más óptima de revisar tales peligros y riesgos puede ser examinar los mismos ejemplos que hemos expuesto en este apartado con el fin de comprobar que, junto a las potencialidades del Sistema 1 que muestran, nos posibilitan descubrir del mismo modo las debilidades del mencionado sistema. Procedemos, por tanto, a dicha revisión.

\section{Las Dificultades Del Sistema 1}

La teoría de la relevancia de Sperber y Wilson (1986) nos ha mostrado que no es muy aconsejable subestimar la función cognitiva del Sistema 1, ya que, gracias a la acción de determinados mecanismos implicados en nuestra dinámica cognitiva, podemos ser más eficientes y eficaces en nuestra acción de conocer la realidad.

Ahora bien, ello no significa que todo lo procedente del Sistema 1 pueda ser tomado sin reservas. Efectivamente, los grandes maestros del ajedrez deben al Sistema 1 la posibilidad de poder enfrentarse a varios adversarios al mismo tiempo. Sin embargo, siempre es posible que, entre tales adversarios, se encuentre un jugador aventajado. Si se diera este caso y el maestro fuera consciente, es obvio que tendría que recurrir a sus capacidades de razonamiento analítico y dejar de proceder de manera automática en función de sus heurísticos del Sistema 1 (dependiendo, claro está, del nivel de juego que impusiera el supuesto adversario aventajado). Esto significa que, en último término, el maestro no puede ignorar totalmente su Sistema 2 ni cuando disputa partidas con aficionados, pues, entre éstos, puede haber alguno que realice sus movimientos de un modo profesional 
o casi profesional y que, para vencerle, sea preciso emplear mayores esfuerzos de análisis. En definitiva, el maestro debe permanecer siempre alerta y, posiblemente, para permanecer alerta es necesaria la acción del Sistema 2.

De la misma manera, hemos de reconocer que, a pesar de que, para los médicos, puede resultar más rápido y dinámico ignorar a pacientes con riesgo de ataque cardiaco que no presentan indicios de enfermedad coronaria alguna, lo cual puede dar lugar, por ejemplo, a considerables reducciones del tiempo de espera de otros pacientes, sabemos que el ataque cardiaco puede darse sin enfermedad coronaria preexistente (Reyna et al., 2003). Esto representa un importante riesgo, puesto que un facultativo que no preste atención al posible ataque cardiaco de un paciente por el hecho de no presentar síntomas asociables con una enfermedad coronaria puede incurrir en una negligencia grave con consecuencias tanto para él (al fin y al cabo, es el responsable de la negligencia) como para el paciente y sus familiares.

Por su parte, con respecto al problema de Linda, creemos que hay algo que decir de un modo categórico: la alternativa (A) del apartado anterior es la que debería ser elegida en primer lugar. Considerar que la opción (B) es más probable que la (A) es un error manifiesto. Esto se ve claramente si, simplemente, pensamos que en la opción (A) no se dice que Linda no sea una activista feminista. Queda abierta la opción de que lo sea o no, y satisfacer una condición siempre es más probable que satisfacer dos condiciones. Reflexionemos, por ejemplo, sobre la probabilidad que existe de que en un curso encontremos a un alumno con ropa blanca. Sea tal probabilidad la que sea, lo cierto es que será mayor que la probabilidad de encontrar, en ese mismo curso, a un alumno con ropa blanca y con zapatos rojos, pues, en este último caso, son dos los requisitos que es preciso cumplir y en el primer caso sólo uno. Por tanto, siempre es necesaria la labor del Sistema 2 revisando y examinando si las intuiciones del Sistema 1 son correctas o no, ya que, eventualmente, podemos cometer errores de cierta importancia si nos guiamos exclusivamente por el heurístico de la representatividad y atendemos únicamente a las características que se pueden relacionar mejor con una circunstancia determinada, y no, por ejemplo, al ámbito lógico, matemático o analítico.

A nuestro juicio, en el párrafo precedente puede encontrarse la clave de toda esta problemática. Es preciso, como hemos visto, aceptar que el Sistema 1 desempeña un rol relevante en el conocimiento humano y que nos ofrece enormes ventajas para minimizar los tiempos y para disminuir los esfuerzos. No obstante, es necesario reconocer también que, como proponen Pérez et al. (2009), es bastante inexacto y que, con frecuencia, conduce a errores. Pero el que reconozcamos las debilidades del Sistema 1 no debe provocar que propugnemos su rechazo, su abolición o su sustitución por el Sistema 2, que es lo que parecen defender Pérez y sus colaboradores. Las intuiciones del Sistema 1 pueden llegar a ser de suma trascendencia para el avance y el progreso del conocimiento y de la ciencia, puesto que, en muchas ocasiones, simplifican la labor de indagación. Lo único que necesitamos es que la actividad del Sistema 1 sea continua y sistemáticamente 
revisada y controlada por el Sistema 2, algo que, en nuestra opinión, como hemos apuntado, aceptarían también Pérez et al.

Esta revisión y este control son realizados por el Sistema 2 en virtud de su propia naturaleza, ya que, como dijimos, los teóricos del razonamiento dual le atribuyen, entre otras características, al Sistema 2 la posibilidad de examinar los resultados logrados mediante el Sistema 1. Por tanto, puede que lo único que haya que evitar sea una insistencia excesiva en considerar a los dos sistemas de la teoría dual como dos compartimentos estancos sin conexión entre sí, o, lo que es lo mismo, en pensar que el aprendizaje pragmático por asociación y el comprensivo son dos tipos exclusivos de aprendizaje incompatibles. Quizás el aprendizaje adecuado para los estudiantes sea un aprendizaje asociativo revisado por un aprendizaje comprensivo y, de esta manera, no sea pertinente obviar al aprendizaje por asociación. Lo que habría que hacer, probablemente, es concederle más protagonismo al aprendizaje comprensivo, pero sin abandonar nunca al asociativo. En este sentido, puede ser muy ilustrativa la división tripartita que, para la teoría dual, se ha establecido recientemente en Stanovich (2012), trabajo en el que se abandonan los términos habituales y se prefiere la denominación Procesos de Tipo 1 para referirse al Sistema 1 y la denominación procesos de Tipo 2 para referirse al Sistema 2. No obstante, lo más relevante para este asunto, a nuestro juicio, es que Stanovich (2012) afirma que, dentro del Sistema 2 (o de los procesos de Tipo 2) es preciso distinguir dos ámbitos: el relativo a las operaciones puramente basadas en algoritmos y el relativo a las disposiciones de pensamiento. Entre estas últimas, incluye, por ejemplo, la necesidad de revisar varias veces un proceso para tener total seguridad acerca de su conclusión o la de recabar toda la evidencia disponible antes de aceptar una opinión. Son, desde su punto de vista, las disposiciones de pensamiento las que hacen distintas cognitivamente a las personas y son éstas también las responsables de que en unas ocasiones se decida comprobar, para, si es necesario, rectificar, la acción del Sistema 1 (o de los procesos de Tipo 1) y en otras no. Quizás la solución a esta problemática resida en que la educación preste una mayor atención a estas disposiciones de pensamiento.

\section{CONClusiones}

Un problema académico de envergadura desde la época moderna bien puede ser el de la incomunicación entre los distintos ámbitos del saber. No cabe duda de que, si entre los investigadores de la pedagogía y los de la ciencia cognitiva existiera un mayor contacto intelectual, podrían alcanzarse construcciones teóricas que recogieran los resultados de ambas áreas y, en nuestro caso concreto, podría notarse con evidencia que los diferentes tipos de aprendizaje pueden corresponder a distintos sistemas cognitivos. Y es que parece que los planteamientos pedagógicos de Pérez y sus colaboradores son, como hemos tratado de mostrar en estas páginas, consistentes con las últimas propuestas de los científicos cognitivos, pues, 
aparentemente, es obvio que el aprendizaje asociativo se encuentra vinculado con el Sistema 1 y que el aprendizaje comprensivo corresponde al Sistema 2.

Empero, más allá de estos vínculos y de estas correspondencias, pensamos que propuestas como la de Pérez y sus colaboradores sólo pueden ser aceptadas si se matizan algunas de sus tesis y se revisan algunos de sus supuestos. Es vital entender correctamente qué es lo que de verdad se está defendiendo cuando se proclama la primacía del aprendizaje comprensivo sobre el aprendizaje asociativo y se argumenta que la función esencial de las instituciones educativas debe ser sustituir el segundo por el primero. Un planteamiento como éste puede interpretarse, desde el punto de vista de la ciencia cognitiva, como un enfoque que supone una superioridad obvia del Sistema 2 y que propone fomentar éste y obviar al Sistema 1.

Como hemos insinuado más arriba, esto puede traducirse en un reduccionismo que sólo concibe como valiosas para la actividad cognoscitiva las destrezas y capacidades características del Sistema 2 y que desdeña los heurísticos y las intuiciones del Sistema 1. Un reduccionismo de esta índole, a la luz de los argumentos expuestos en este trabajo, no parece nada recomendable. Lo más oportuno puede ser admitir que los dos sistemas de razonamiento de los que nos habla la teoría dual ofrecen ventajas y potencialidades, pues parece evidente que el Sistema 1 también tiene su función cognitiva y que sólo necesita ser controlado y revisado por medio de las capacidades analíticas del Sistema 2, necesidad, esta última, con la que, al fin y al cabo, también estarían de acuerdo Pérez y sus colaboradores. Y es que no nos podemos olvidar de todos los aspectos positivos que acompañan al Sistema $1 \mathrm{y}$, por lo tanto, al aprendizaje por asociación.

No nos gustaría finalizar, no obstante, sin hacer mención a lo beneficioso que podría ser para cualquier docente de cualquier nivel educativo tener conocimiento de las teorías contemporáneas de la ciencia cognitiva. Si el profesorado fuera consciente de la posible existencia del Sistema 1 y del Sistema 2, podría abrirse un interesante camino para la metacognición, actividad que tan importante es, como lo reconocen también Pérez et al., para lograr un aprendizaje adecuado. Para nosotros, es indiscutible que, si un profesor conoce correctamente las características de cada uno de los dos sistemas de la teoría dual de razonamiento, puede, por ejemplo, diseñar actividades y pensar en recursos metodológicos atendiendo al sistema sobre el que pretende actuar o intervenir, lo cual puede favorecer el éxito de su práctica pedagógica. Sin embargo, el conocimiento de los dos sistemas es también muy apropiado para el estudiante, pues, al comprender cuáles de sus capacidades están operando en una situación concreta de aprendizaje, puede llegar a ser, en mayor medida, partícipe de su propia educación y protagonista del proceso personal mediante el que aprende.

Vemos así, nuevamente, lo positivo que podría resultar un mayor acercamiento entre los diferentes ámbitos del saber, acercamiento que, claro está, beneficiaría a todas las partes y que redundaría en un mayor desarrollo de tales ámbitos. Probablemente, la ciencia cognitiva tiene mucho que decir sobre temáticas pedagógicas y la práctica pedagógica dispone de muchas evidencias de las que 
EL APRENDIZAJE EN LA UNIVERSIDAD Y LA TEORÍA DEL PROCESO DUAL DE RAZONAMIENTO

informar a la ciencia cognitiva. Es el momento, pues, de instaurar una comunicación más fluida.

\section{REFERENCIAS BIBLIOGRÁFICAS}

Beth, E. W. y Piaget, J. (1966) Mathematical epistemology and psychology. Dordrecht, Reidel.

ELLIS, N. C. (2011) Implicit and explicit SLA and their interface, en SANZ, C. y LEOw, R. P. (eds.) Implicit and explicit language learning. Conditions, processes, and knowledge in SLA and bilingualism. Washington D. C., Georgetown University Press, 35-47.

EPSTEIN, S. (1994) Integration of the cognitive and psychodynamic unconscious. American Psychologist, 49, 709-724.

Evans, J. St. B. T. (1989) Bias in human reasoning: causes and consequences. Brighton, Erlbaum.

- (2006) The heuristic-analytic theory of reasoning: extension and evaluation. Psychonomic Bulletin E Review, 13 (3), 378-395.

- (2008) Dual-processing accounts of reasoning, judgment, and social cognition. Annual Review of Psychology, 59, 255-278.

Hempel, C. G. (1945) Studies in the logic of confirmation (I). Mind, 54, 1-26.

INGLIS, M. y Simpson, A. (2006) Characterising mathematical reasoning: studies with the Wason selection task, en Bosch, M. (ed.) Proceedings of the Fourth Congress of the European Society for Research in Mathematics Education. Sant Feliu de Guíxols, 17681777.

INHELDER, B. y PIAGET, J. (1958) The growth of logical thinking from childhood to adolescence. Nueva York, Basics Books.

Lave, J. y Wenger, E. (1991) Situated learning. Cambridge, Cambridge University Press.

López, M. (2008) La paradoja de Hempel. Ciencia Cognitiva, 2 (3), 94-95.

- (2009a) ¿Funciona el cerebro de los grandes maestros de ajedrez de manera diferente al de la población general? Ciencia Cognitiva, 3 (3), 83-85.

- (2009b) El problema de Linda y la falacia de la conjunción. Konvergencias. Filosofía y Culturas en Diálogo, 7 (21), 24-43.

- (2011) La falacia de la conjunción y la contextualización en el autismo. Estudios Pedagógicos, 37 (1), 279-291.

Pérez, A.; Soto, E.; Sola, M. y Serván, M. J. (2009) Aprender cómo aprender. Autonomía y responsabilidad: el aprendizaje de los estudiantes. Madrid, Akal.

REBER, A. (1993) Implicit learning and tacit knowledge. Nueva York, Oxford University Press.

REYNA, V. F. (2004) How people make decisions that involve risk: a dual-processes approach. Current Directions in Psychological Science, 13, 60-66.

ReYNA, V. F. y ADAm, M. B. (2003) Fuzzy-trace theory, risk communication, and product labeling in sexually transmitted diseases. Risk Analysis, 23, 325-342.

Reyna, V. F.; LLOYd, F. J. y BRAINerd, C. J. (2003) Memory, development, and rationality: an integrative theory of judgment and decision-making, en SCHNEIDER, S. y SHANTEAU, J. (eds.) Emerging perspectives on judgment and decision research. Nueva York, Cambridge University Press, 201-245.

SCHNEIDER, W. y SHIFFrin, R. M. (1977) Controlled and automatic human information processing I: detection, search and attention. Psychological Review, 84, 1-66. 
EL APRENDIZAJE EN LA UNIVERSIDAD Y LA TEORÍA DEL PROCESO DUAL DE RAZONAMIENTO

Sloman, S. A. (1996) The empirical case for two systems of reasoning. Psychological Bulletin, 119, 3-22.

SPERBER, D. y WiLSON, D. (1986) Relevance: communication and cognition. Oxford, Blackwell.

STANOVICH, K. E. (1999) Who is rational? Studies of individual differences in reasoning. Mahwah, Erlbaum.

- (2004) The robot's rebellion: finding meaning in the age of Darwin. Chicago, Chicago University Press.

- (2012) On the distinction between rationality and intelligence for understanding individual differences in reasoning, en HOLYOAK, K. y MORRISON, R. (eds.) The oxford handbook of thinking and reasoning. Nueva York, Oxford University Press, 343-365.

Tversky, A. y Kahneman, D. (1983) Extensional vs. intuitive reasoning: the conjunction fallacy in probability judgment. Psychological Review, 90, 293-315.

WASSERman, E. A. y MilLeR, R. R. (1997) What's elementary about associative learning? Annual Review of Psychology, 45, 573-607.

Wilson, T. D. (2002) Strangers to ourselves. Cambridge, Belknap. 